\title{
SOBRE A REVERSÃO DA FIBROSE HEPÁTICA ESQUISTOSSOMÓTICA APÓS TERAPÊUTICA ESPECÍFICA. ESTUDO HISTOLÓGICO
}

\author{
Edmundo Chapadeiro e Luiz Claro Pitanga
}

\begin{abstract}
Biópsias cirúrgicas de fígado de portadores da forma bepatesplênica compensada da esquistossomose mansônica, previamente tratados com oxaminiquine (Mansil) $e$ submetidos, posteriormente, à anastomose esplenorrenal, foram estudadas através de algumas técnicas histológicas e imunobistoquímicas. Embora a pesquisa de vermes adultos, de ovos e/ou de granulomas fosse negativa, em todos os espécimes examinados, a fibrose portal estava presente.
\end{abstract}

Palavras-chaves: Esquistossomose mansônica. Forma hepatesplênica. Fibrose bepática. Reversão. Oxaminiquine.

A reversão da fibrose hepática esquistossomótica, após tratamento específico com oxaminiquine é relatada por vários pesquisadores em nosso meio ${ }^{2}$. Embora outros ${ }^{4}$, tenham admitido a reabsorção da fibrose na maioria dos casos verificaram que, em alguns, ela pode permanecer inalterada ou mesmo agravar-se.

Visto que são desconhecidas as razões para a não reversão ou a reversão parcial da fibrose, pareceu-nos de interesse relatar o que ocorre no fígado de portadores da forma hepatesplênica da esquistossomose, que apresentaram sangramento (hematêmese e/ou melena), após terapêutica específica com a oxaminiquine.

\section{MATERIAL E MÉTODOS}

Fragmentos de fígado de 12 , dentre 30 portadores da forma hepatesplêmica compensada da esquistossomose mansônica, foram retirados durante anastomose esplenorrenal seletiva, no período de 1985 a 1991 no Hospital São Vicente de Paula (Governador Valadares, MG), em conseqüência de sangramento de varizes esofágicas e/ou melena. Os 18 casos restantes não foram incluídos neste estudo por falta de

Departamento de Patologia, Medicina Legal e do Curso de Pós-graduação em Patologia Humana da Faculdade de Medicina do Triângulo Mineiro, Uberaba, MG.

Endereço para correspondência: Prof. Edmundo Chapadeiro. Curso de Pós-graduação em Patologia Humana/FMTM. Av. Getúlio Guaritá 130, 38025-440 Uberaba, MG, Brasil. Fone: (034) 312-7722 - r: 1154; Fax: (034) 312-6640. Recebido para publicação em 22/03/96. dados precisos sobre o tempo decorrido entre a terapêutica clínica e a cirurgia.

A idade dos pacientes variou entre 18 e 72 anos; oito eram homens e quatro mulheres; cinco eram brancos e sete pardos. Nenhum deles apresentou quaisquer sintomas e/ou sinais de outra doença hepática (hepatite; cirrose, etc). Todos os pacientes receberam oxaminiquine (Mansil) na dose recomendada, pelo menos um ano antes da cirurgia e a maior parte deles permaneceu na área endêmica. A determinação de HbSAg no soro foi negativa em todos os casos. Os exames coprológicos foram negativos para ovos do S. mansoni em todos os indivíduos antes do ato cirúrgico.

Os fragmentos de fígado obtidos durante o ato operatório tinham em média $0,8 \times 1,5 \mathrm{~cm}$ e foram fixados em formol a 10\%, após secção em duas metades; uma foi incluída em parafina para o estudo rotineiro e a outra utilizada para cortes em congelação e coloração para lipídios (Sudão Negro). Secções em parafina de 5-6m foram coradas pela hematoxilina-eosina (HE), tricrômico de Heidenhain e pela fucsina de Weigert para o elástico; também foram utilizados o Sírius-red para o colágeno e o teste de Shikata para detecção do HbsAg; a pesquisa dos antígenos totais do S. mansoni foi realizada em oito casos pela técnica da streptavidina-biotina.

\section{RESULTADOS}

Os principais dados dos pacientes com a forma hepatesplênica da esquistossomose, 
Chapadeiro E, Pitanga LC. Sobre a reversão da fibrose hepática esquistossomótica após terapêutica específica. Estudo histológico. Revista da Sociedade Brasileira de Medicina Tropical 30:53-56, jan-fev, 1997.

estudados e tratados com oxaminiquine, encontram-se resumidos na Tabela 1.

O estudo histológico rotineiro do fígado mostrou que nos doze pacientes, a lesão era fundamentalmente a mesma tanto no sexo masculino quanto no feminino, assim como em jovens e adultos (Tabela 2).

Havia alargamento dos espaços portobiliares em conseqüência da peripileflebite crônica, acompanhada de intensa proliferação de

Tabela 1 - Portadores da forma hepatesplênica da esquistossomose mansônica tratados com oxaminiquine* e submetidos à anastomose esplenorrenal seletiva.

\begin{tabular}{|c|c|c|c|c|c|c|}
\hline № & Identificação & Sexo & Cor & Idade (ano) & Sintomatologia & Cirurgia (ano) \\
\hline 1 & AJS & M & $\mathrm{P}$ & 72 & hepatesplenomegalia, hematêmese, melena & 3 \\
\hline 2 & NLS & $\mathrm{F}$ & $\mathrm{P}$ & 33 & hepatesplenomegalia, hematêmese, melena & 1 \\
\hline 3 & NEC & M & $\mathrm{P}$ & 66 & hepatesplenomegalia, hematêmese, melena & 5 \\
\hline 4 & DLV & M & B & 30 & hepatesplenomegalia, melena & 2 \\
\hline 5 & DJA & M & $\mathrm{B}$ & 23 & hepatesplenomegalia, hematemese & 5 \\
\hline 6 & LPS & $\mathrm{F}$ & $\mathrm{P}$ & 36 & esplenomegalia & 4 \\
\hline 7 & $\mathrm{JCN}$ & M & B & 47 & hematêmese, melena & 10 \\
\hline 8 & ACC & M & $\mathrm{B}$ & 25 & hepatesplenomegalia, hematêmese, melena & 2 \\
\hline 9 & PDN & M & $\mathrm{P}$ & 25 & hematêmese, melena & 4 \\
\hline 10 & TDOF & $\mathrm{F}$ & $\mathrm{P}$ & 28 & melena & 3 \\
\hline 11 & LAR & $\mathrm{F}$ & B & 18 & hematêmese, melena & 4 \\
\hline 12 & JFR & M & $\mathrm{P}$ & 62 & melena & 2 \\
\hline
\end{tabular}

Tabela 2 - Sumário das lesões hepáticas de portadores da forma hepatesplênica da esquistossomose mansônica submetidos a anastomose esplenorrenal seletiva, após tratamento com oxaminiquine*

\begin{tabular}{|c|c|c|c|c|c|c|c|c|c|c|c|}
\hline Identificação & $\begin{array}{l}\text { Granulomas } \\
\text { em atividade }\end{array}$ & $\begin{array}{c}\text { Granulomas } \\
\text { cicatriciais }\end{array}$ & $\begin{array}{c}\text { Ovos } \\
\text { viáveis }\end{array}$ & $\begin{array}{l}\text { Ovos } \\
\text { mortos }\end{array}$ & $\begin{array}{c}\text { Lesões ramos } \\
\text { portais }\end{array}$ & $\begin{array}{c}\text { Fibrose } \\
\text { portal }\end{array}$ & $\begin{array}{l}\text { Infiltrado } \\
\text { portal }\end{array}$ & Angiomatóides & $\begin{array}{c}\text { Lesões } \\
\text { arteriais }\end{array}$ & $\begin{array}{l}\text { Neoformação } \\
\text { dutos biliares }\end{array}$ & $\begin{array}{l}\text { Fibrose } \\
\text { capsular }\end{array}$ \\
\hline$\overline{\mathrm{AIS}}$ & 0 & + & 0 & 0 & +++ & +++ & ++ & +++ & +++ & +++ & +++ \\
\hline NLS & 0 & 0 & 0 & 0 & ++ & ++ & ++ & ++ & + & + & + \\
\hline NEC & 0 & 0 & 0 & 0 & ++ & ++ & ++ & ++ & ++ & ++ & + \\
\hline DLV & 0 & 0 & 0 & 0 & ++ & ++ & ++ & ++ & ++ & +++ & + \\
\hline DJA & 0 & 0 & 0 & 0 & ++ & +++ & + & ++ & ++ & ++ & + \\
\hline LPS & 0 & 0 & 0 & 0 & + & ++ & ++ & ++ & + & ++ & + \\
\hline $\mathrm{JCN}$ & 0 & 0 & 0 & 0 & ++ & + & ++ & + & - & + & + \\
\hline $\mathrm{MC}$ & 0 & 0 & 0 & 0 & ++++ & +++ & ++ & +++ & +++ & +++ & + \\
\hline ACC & 0 & 0 & 0 & + & ++ & +++ & ++ & ++ & + & + & ++ \\
\hline PND & 0 & 0 & 0 & 0 & ++ & +++ & + & ++ & ++ & ++ & + \\
\hline TDOF & 0 & 0 & 0 & 0 & +++ & +++ & ++ & ++ & ++ & +++ & ++ \\
\hline LAR & 0 & 0 & 0 & 0 & ++ & ++ & + & ++ & + & + & + \\
\hline JFR & 0 & 0 & 0 & 0 & ++ & +++ & + & ++ & ++ & + & ++ \\
\hline
\end{tabular}

tecido conjuntivo, dilatação angiomatóide dos ramos portais e neoformação de dutos biliares, sem subversão da arquitetura lobular (Figura 1).

O conjuntivo neoformado é composto principalmente de células com a morfologia dos fibroblastos, entre os quais se entremeiam células fusiformes com a mesma afinidade tintorial pelo tricrômico, daquelas da média dos ramos portais e dos feixes colágenos. Estes são geralmente muito densos, às vezes de aspecto hialinizado, entrecruzando-se com feixes mais delgados; com freqüência entre as áreas mais densas, predominantes, aparecem áreas mais frouxas (periferia dos lóbulos e áreas septais). Os feixes colágenos densos mostram intensa coloração vermelho-alaranjada, à microscopia de polarização, enquanto os feixes delgados exibem polarização amarela ou amarelo-esverdeada (Figura 2). Entre os feixes colágenos, fibras elásticas mostram-se abundantes, espessadas rôtas, formando grumos hipercorados. Fibras reticulares (colágeno I) são também abundantes, no conjuntivo neoformado mais frouxo (áreas septais) e na periferia dos lóbulos. No conjuntivo frouxo e, de permeio aos elementos citados, observa-se infiltrado celular, de intensidade variável, constituído especialmente por mononucleares (macrófagos, linfócitos e plasmócitos) e 
Chapadeiro E, Pitanga LC. Sobre a reversão da fibrose hepática esquistossomótica após terapêutica específica. Estudo bistológico. Revista da Sociedade Brasileira de Medicina Tropical 30:53-56, jan-fev, 1997.

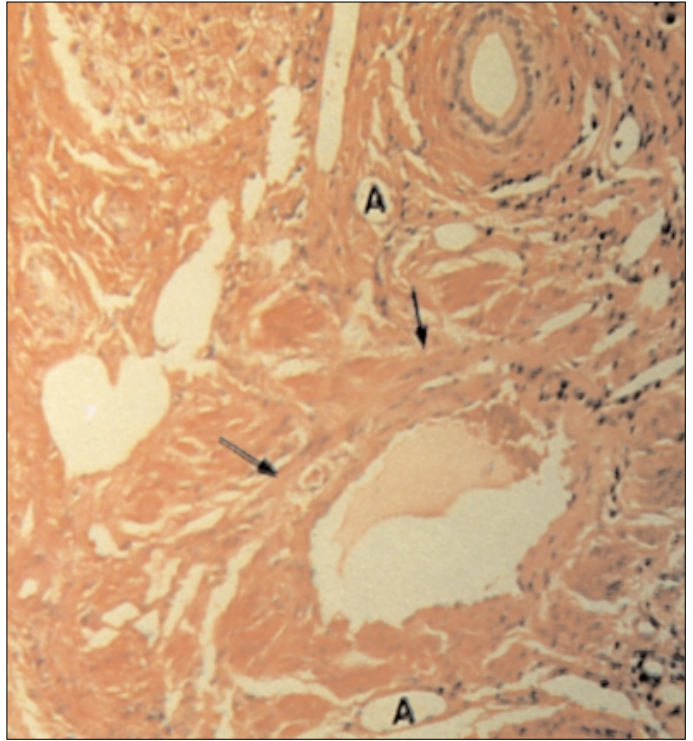

Figura 1 - Biópsia bepática de paciente de 23 anos, tratado com oxaminiquine cinco anos antes da anastomose esplenorrenal. Espaço portal alargado por neoformação conjuntiva $e$ dilataç̃o angiomatóide (A); atrofia $e$ fibrose parcial da musculatura de ramo portal (setas). Ausência de ovos e granulomas. HE $x 100$.

granulócitos, às vezes, com predomínio de eosinófilos. A dilatação angiomatóide está sempre presente, nas áreas portais, porém com intensidade variável.

Vermes adultos ou restos destes, ovos viáveis, assim como granulomas em atividade não foram observados; o pigmento esquistossomótico era em geral ausente. O teste imunohistoquímico para antígenos totais do $S$. mansoni foi negativo em 8 dos casos em que foi realizado; a reação de Shikata foi negativa nas secções de todos os casos.

Os hepatócitos apresentam-se ora de aspecto normal, ora com graus variáveis de atrofia, degeneração (vacuolar, granulosa, esteatose) ou ainda com sinais de poliploidia.

A cápsula de Glisson, mostra alternadamente áreas espessadas por intensa neoformação conjuntiva, com as características já citadas, e áreas não espessadas.

Nos 18 casos restantes, nos quais não se pôde precisar o tempo decorrido entre a terapêutica específica e a cirurgia, o quadro histológico era fundamentalmente o mesmo.

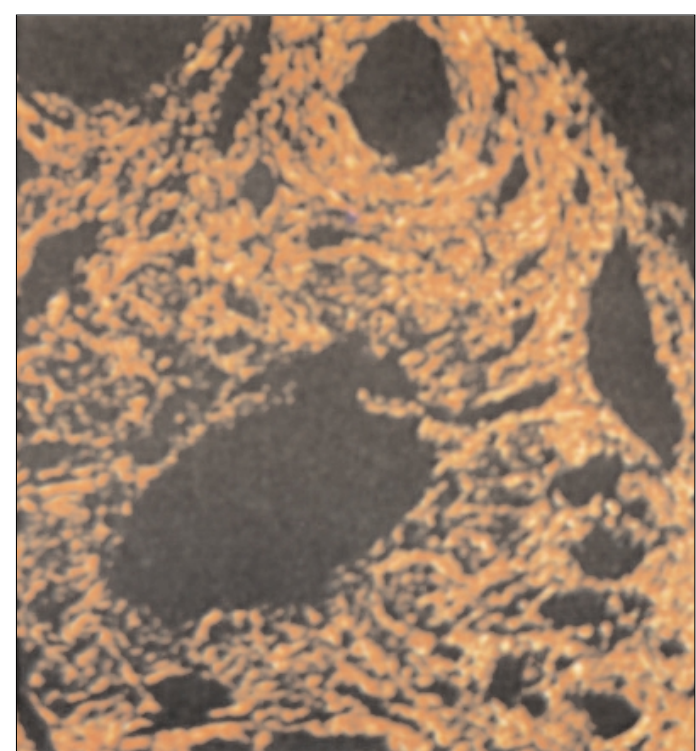

Figura 2 - Mesmo campo da figura anterior mostrando abundante colágeno do tipo III (vermelbo-alaranjado) e escasso do tipo I (amarelo-esverdeado). Sirius-red Polarização $\times 100$

\section{DISCUSSÃO}

Posto que não conhecéssemos o quadro histológico do fígado de nossos pacientes anteriormente à terapêutica específica, a ausência de vermes, de ovos viáveis e/ou de granulomas em atividade, assim como a reação negativa para antígenos totais do $S$. mansoni na lesão hepática, parecem comprovar a eficácia da terapêutica; nestes casos entretanto esta não foi capaz de reverter a fibrose portal mesmo dez anos após o tratamento. Nossa casuística poderia constituir, portanto, aquela parcela de pacientes que, mesmo tratados, não mostram regressão significativa da fibrose hepática ${ }^{3}$; os focos de rarefação no conjuntivo neoformado descritos à microscopia óptica e eletrônica ${ }^{1}$ eram escassos e de pequena monta.

Em nosso material, os achados histológicos não estavam associados à presença de quaisquer lesões atribuíveis a outras hepatopatias; de outro lado, confirmam aqueles ${ }^{4}$, segundo os quais a idade dos pacientes, assim como a duração da infecção, não parecem suficientes para explicar a reversão das lesões hepáticas, 
Chapadeiro E, Pitanga LC. Sobre a reversão da fibrose hepática esquistossomótica após terapêutica específica. Estudo histológico. Revista da Sociedade Brasileira de Medicina Tropical 30:53-56, jan-fev, 1997.

embora, em crianças maiores de nove anos tenha sido verificada ${ }^{3}$ melhor resposta à terapêutica. Entretanto relatos baseados na ultrasonografia tem mostrado também irreversibilidade das lesões hepáticas em adolescentes submetidos à terapêutica específica ${ }^{4}$.

Ainda que fatores genéticos e/ou raciais sejam considerados também ${ }^{5}$ relacionados com a gravidade e com a resposta terapêutica das lesões hepáticas, em nossos casos, a persistência da fibrose ocorreu de modo igual, praticamente, em brancos e em não brancos (pardos). O fato da fibrose de Symmers ocorrer apenas em 5\%, aproximadamente, dos indivíduos de uma área endêmica, parece indicar a importância da resposta individual na síntese e/ou degradação do colágeno.

Finalmente, como é sabido, a neoformação colágena nos espaços portais não constitui o único elemento da lesão de Symmers e nem a única causa do distúrbio hemodinâmico da forma hepatesplênica: a obstrução, a deformidade, a perda parcial da musculatura e da camada elástica dos ramos portais, assim como as lesões arteriais, persistiram também após a terapêutica.

\section{SUMMARY}

Surgical liver biopsies of patients of both sexes, between 18 and 72 years old, with the compensated hepatoesplenic form of schistomiasis mansoni, previously treated with oxamniquine (Mansil) were studied by histological and immunohistochemical methods. Although the search for parasites and/or granulomas was negative in all specimen studied, the portal fibrosis remained.

Key-words: Schistosomiasis mansoni. Hepatesplenic form. Symmers' fibrosis. Reversion. Oxamniquine.

\section{AGRADECIMENTOS}

À Prof ${ }^{\underline{a}} \operatorname{Dr}^{\underline{a}}$ Maria Irma Seixas Duarte pelas técnicas imunohistoquímicas realizadas; à Srta. Alessandra Scoda e à Sra. Denise Borges e Borges Laterza pela digitação do manuscrito.

\section{REFERÊNCIAS BIBLIOGRÁFICAS}

1 Andrade ZA, Peixoto E, Sylviane G, Grimand JA. Hepatic connective changes in hepatosplenic schistosomiasis. Human Pathology 23:566-573, 1992.

2 Bina JC. Influência da terapêutica específica na prevenção e reversão das formas graves da esquistossomose. Memórias do Instituto Oswaldo Cruz 82 (supl IV):331-332, 1987.

3 Bina JC, Prata A. Regressão da hepatesplenomegalia pelo tratamento específico da esquistossomose. Revista da Sociedade Brasileira de Medicina Tropical 16:213-218, 1983.

4. Dietze R, Prata A. Rate of reversion of hepatosplenic schistosomiasis after specific therapy. Revista da Sociedade Brasileira de Medicina Tropical 19:69-73, 1986.

5. Mohamed-Ali Q, Doenring-Schwerdtfeger E, Abdel-Rahim IM, Schlake J, Kadorff R, Franke D, Kaiser C, Elsheikh M, Abdalla M, Schafer P, Ehrich $\mathrm{JHH}$. Ultrasonographical investigation of periportal fibrosis in children with Schistosoma mansoni infection: reversibility of morbidity seven months after treatment with praziquantel. American Journal of Tropical Medicine and Hygiene 44:444-451, 1990.

6 Phillips SM, Lanimice PJ. Immunopathology of granuloma formation and fibrosis in Schistosomiasis. Pathology Today 2:296-302, 1986.

7 Raso P. Esquistossomose mansônica. In: Brasileiro Filho G, Pittela JEH, Pereira FEL, Bambirra EA, Barbosa AJA (eds) Bogliolo Patologia. $5^{\mathrm{a}}$ edição, Guanabara Koogan Rio de Janeiro p. 1124-1145, 1994.

8 Tavares-Neto J, Prata A. Forma hepatosplênica da esquistossomose mansônica, em relação à composição racial e ao nível sócio-econômico, em Catolândia - Bahia. Revista da Sociedade Brasileira de Medicina Tropical 23:37-42, 1990. 